\title{
TRAÇOS DE PERSONALIDADE NA INFÂNCIA E DISTORÇÃO E INTEGRAÇÃO DE FORMAS: UM ESTUDO DE VALIDADE
}

\author{
Fermino Fernandes Sisto \\ José Maurício Haas Bueno" \\ Fabián Javier Marín Rueda
}

\begin{abstract}
RESUMO. As relações entre traços de personalidade e percepção visomotora foram estudadas em 344 estudantes, de 6 a 12 anos, de ambos os sexos. Com o teste gestáltico de Bender obtiveram-se pontuações para distorção de formas e integração e outras medidas, soma das pontuações das figuras. A Escala de Personalidade para crianças informou sobre neuroticidade, psicoticidade, extroversão e adequação. Houve correlações positivas e significativas em relação a neuroticismo e algumas medidas de integração; psicoticismo com medidas de distorção e uma medida de integração; e algumas medidas discriminaram grupos extremos de neuroticismo e psicoticismo. Observou-se que a diminuição da integração visomotora implicou em aumentou de neuroticidade; e o aumento da pontuação em psicoticidade aumentou da distorção de formas.
\end{abstract}

Palavras-chave: traços de personalidade; teste gestáltico visomotor de Bender; infância.

\section{PERSONALITY TRAITS IN CHILDHOOD, DISTORTION AND INTEGRATION OF FORMS: A VALIDITY STUDY}

\begin{abstract}
The relationships among personality traits and visomotor perception were studied in 384 male and female students, from 6 to 12 years of age. With Bender Test it was possible to obtain the scores sum of the figures, scores of distortion and integration of forms, and other measures. The Personality Scale for Children informed about neuroticism, psychoticism, extroversion and adequacy. Positive and significant correlations were found for neuroticism with integration measures and psychoticism with distortion measures and one integration measure. Some measures categorized extreme groups of neuroticism and psychoticism. It was observed that as diminishing the scores of visomotor integration, the scores of neuroticism increased, and as the psychoticism scores increased, the scores of the distortion of forms also increased.
\end{abstract}

Key words: personality traits; Bender Gestalt Test; childhood.

\section{INTRODUÇÃO}

O teste gestáltico visomotor de Bender foi originalmente construído por Lauretta Bender, em 1938, com o propósito de fornecer uma avaliação psicológica segundo os princípios da teoria gestáltica. Segundo Bender (1938), a qualidade da reprodução das figuras é determinada por fatores biológicos e de ação sensório-motora, que variam em razão do padrão de desenvolvimento e nível maturacional de cada indivíduo e de seu estado patológico funcional e organicamente induzido.
Independentemente do método de aplicação e avaliação, o teste gestáltico visomotor de Bender tem sido muito usado por psicólogos clínicos para a avaliação da personalidade e em avaliações diagnósticas psiquiátricas e médicas. Vários estudos relatam que ele está entre os mais usados (por exemplo, Kamman \& Kram, 1955; Archer, Maruish, Imhof e Piotrowski, 1991).

Seu sistema de avaliação, no entanto, segue uma abordagem evolutiva na análise dos protocolos infantis e um enfoque clínico no caso de protocolos de adultos. A necessidade de um sistema objetivo de avaliação,

\footnotetext{
Docente da Universidade São Francisco, doutor e livre docente.

Endereço para correspondência: Rua Carlos Guimarães, 150, apto. 82, Cambuí, CEP 13024-200, Campinas-SP.

E-mail: ffsisto@hotmail.com

\# Docente da Universidade Mackenzie, mestre.

II Discente de graduação, Universidade São Francisco.
} 
que melhorasse sua validade e precisão, levou vários psicólogos a desenvolverem sistemas de pontuação para esse teste e vários sistemas de avaliação ganharam notoriedade no meio científico. Pascal \& Suttell (1951) fornecem um sistema para estimativa do grau de ajustamento emocional; por sua vez, Clawson (1980) identificou sinais e desvios na reprodução das figuras do Bender como indicadores de perturbação emocional; e também um sistema para avaliação da maturidade neurológica visomotor foi desenvolvido por Koppitz (1964). Mais recentemente, Lacks (1984) construiu um sistema para a triagem de pacientes orgânicos e não orgânicos e alcoolismo; e Hutt (1985) adaptou uma Escala de Psicopatologia para diferenciar indivíduos normais de diversos quadros patológicos.

A validade do Bender, seja qual for o método de utilização em consideração, tem sido bastante questionada e estudada. Há uma vasta literatura citando a utilização ou investigando as propriedades psicométricas do instrumento.

Em relação ao método de Pascal \& Suttell (1951), alguns estudos relatam sua efetividade como instrumento para discriminar entre diferentes psicopatologias. Para citar alguns, Matchabely \& Bertrand (1953) encontraram padrões definidos para vários quadros psicopatológicos; Bambaren-Vigil (1958) afirma sua validade para diferenciar entre pacientes esquizofrênicos e normais e não pacientes; e, estudando psicóticos esquizofrênicos, não psicóticos com desordem de personalidade e estudantes, Stewart \& Cunningham (1958) concluíram que as pontuações aumentaram conforme o grau da patologia aumentou. Outros estudos sugerem parcial ou nenhuma efetividade do método Pascal \& Sutell (por exemplo, Tamkin, 1957).

Também há controvérsias quanto à validade do método de análise configuracional de Hutt (1985). Foi encontrada validade discriminativa para sua utilização com pacientes esquizofrênicos, depressivos e lesionados cerebrais (Lownsdale, Rogers e McCall, 1989) e para organicidade (Brilliant \& Gynther, 1963). Encontraram-se também correlações estatisticamente significativas entre indicadores de hostilidade e escalas de agressividade, proporcionando algum apoio para sua utilização em avaliação da personalidade (Sangster, Rogers e Searight, 1993). Ao lado disso, foram estudadas as propriedades psicométricas e a utilidade clínica das escalas de psicopatologia e percepção "adience-abience" da adaptação Hutt (Wasserman, 1995), e os resultados não apóiam uma validade de construto.

Não foram muitos os pesquisadores que se preocuparam com a utilização do Bender em crianças.
Porterfield (1969) encontrou diferenças nos escores do Bender entre gagos e um grupo mal-adaptado, sugerindo sua relevância. Também Handler \& McIntosh (1971) relatam que o Bender e o desenho da figura humana foram mais eficientes do que observação comportamental e auto-relato, na identificação da agressividade. Ao lado disso, comparando delinqüentes, crianças normais e com distúrbios, Hutt, Dates e Reid (1977) encontraram que as diferenças não ssuficientemente altas para garantir seu uso para propósitos preditivos individuais. E, ainda, Rossini (1993) avaliou a validade concorrente da Escala de Psicopatologia do Bender entre crianças com desvio de ajustamento e desvio de conduta nãoclínicas, e verificou ter havido diferenças significativas entre os grupos.

Há um grupo de pesquisadores que procurou correlações entre distorções ou sinais na reprodução das figuras do Bender e características ou traços de personalidade. Murray \& Roberts (1956) relataram os resultados para a validade do Bender como medida de personalidade restrita e expansiva. A hipótese de que a distorção do Bender esteja relacionada com os conteúdos e determinantes do Rorschach foi defendida por Clawson (1980). Por sua vez, Gavales \& Millon (1960) informaram que estudantes com altas pontuações na escala de ansiedade tenderam significativamente para um tamanho menor no desenho. Ao estudar pacientes com constrição em seus registros antigos do Bender, Johnson (1973) observou diferença significativa nos escores de depressão, comparados com indicação recente de redução. Ao lado disso, Holmes, Dungan e Medlin (1984) verificaram associação entre traços de personalidade e estilos de desenhos em pacientes com dificuldades de fechamento e com tendência às margens. Ao lado disso, Wallbrown, Wirth e Engin (1975), investigando a relação entre erros (laços, angulação, círculos e pontos) e impulsividade, concluíram que os erros não podem ser explicados pela impulsividade; porém, Brannigan, Barone e Margolis (1978) realizaram a mesma investigação e encontraram relação significativa entre eles.

Existem pesquisas com resultados que levantam mais dúvidas sobre esse tipo de relação. Explorando em que extensão a constrição (redução) é uma característica geral da personalidade, Wohl (1957) indicou que o conceito possui pouca generalização. Ao lado disso, Prado, Peyman \& Lacey (1960) concluíram que o teste Bender, por meio de curvas achatadas e/ou angulação, não diferenciou psicóticos com e sem afeto embotado. Por sua vez, Sternberg \& Levine (1965) estudaram a "penetração" do desenho 6 
no desenho 5 em pacientes psiquiátricos, sem concluir que o estilo de desenho no Bender sirva como base para inferir traços de personalidade. E estudando usuários de heroína, Korin (1974) encontrou que o uso constrito do espaço, tendência à rotação dos desenhos e perseverações foram significativamente mais freqüentes em usuários, embora as diferenças entre os escores totais de pacientes desintoxicados e nãodesintoxicados pudessem ser atribuídas ao acaso.

Nesse contexto, não de buscar nova escala de pontuação, mas de estudar particularidades do teste, é que se insere este estudo. Observou-se na literatura uma tendência a estudar o tamanho dos desenhos e do espaço ocupado, como também das curvas e achatamentos dos desenhos. Também parece haver uma tendência maior a estudar o Bender em relação a patologias. Em contraposição, optou-se por estudar traços de personalidade em crianças sem histórico clínico, em relação à escala de maturação neurológica (Koppitz, 1964). Mais especificamente, das quatro categorias de desvios na reprodução das figuras foram estudadas, nesta pesquisa, a distorção de formas e a falta de integração nas figuras em relação a traços de personalidade, tanto para verificar correlações como para avaliar a possibilidade discriminativa de grupos extremos.

\section{MÉTODO}

\section{Participantes}

Foram sujeitos da pesquisa 344 crianças do sexo masculino e do sexo feminino, de $1^{\underline{a}}$ a $4^{\underline{a}}$ séries de uma escola pública, com idades variando entre 6 e 12 anos (média 8,8 anos e desvio padrão de 1,31).

\section{Instrumento}

\section{Teste gestáltico visomotor de Bender}

O Teste gestáltico visomotor de Bender foi construído para fornecer um índice de maturação perceptomotora. Consiste de nove figuras (A, 1, 2, 3, 4, 5, 6, 7 e 8), que são apresentadas uma a uma, para serem copiadas pelo sujeito em uma folha em branco, sem auxílio mecânico.

Neste estudo, optou-se pelo critério de Koppitz, segundo o qual avaliam-se quatro quesitos: distorção da forma pelas figuras $\mathrm{A}, 1,3,5,6,7$ e 8 ; rotação pelas figuras A, 1, 2, 3, 4, 5, 7 e 8; integração pelas figuras A, 2, 3, 4, 5, 6 e 7; e perseveração pelas figuras 1,2 e 6 . Para este trabalho interessaram apenas os itens referentes à distorção e integração da forma nas figuras A, 1, 2, 3, 4, 5 e 7 .
Conforme os critérios propostos por Koppitz, para cada erro cometido pela criança foi atribuído um ponto. A avaliação foi realizada por um grupo de seis pessoas, que concordaram entre si na atribuição de cada ponto aos erros cometidos pelos sujeitos.

Além da correção por item, foram criadas outras medidas, produtos da soma de itens, a saber: distorção de forma, somatório dos pontos atribuídos às figuras analisadas nesse quesito; integração de forma, somatório dos pontos atribuídos às figuras analisadas nesse quesito; pontuação geral, somatório das medidas anteriores; distorção nas figuras geométricas A e 7; distorção nas figuras geométricas 1, 3 e 5; distorção de forma ponderada, somatório do total de pontos em distorção nas figuras geométricas A e 7 e um terço dos pontos em distorção nas figuras geométricas 1, 3 e 5; integração acentuada, somatório das figuras A, 3, 43 7, cuja falta de integração foi observada em pelo menos $9 \%$ dos sujeitos.

\section{Traços de Personalidade}

A avaliação dos traços de personalidade foi realizada por meio da Escala de Traços de Personalidade para Crianças ${ }^{1}$, um questionário com 30 itens (Sisto, 1998), com precisão e validade para os seguintes fatores: neuroticismo, psicoticismo, extroversão e adequação. É um instrumento para ser usado em crianças de 5 a 10 anos de idade.

A validade fatorial dessa escala foi analisada em um grupo de 2538 crianças de 5 a 10 anos de idade $^{2}$, com os quatro fatores explicando 49,294 da variância. Os coeficientes de consistência interna (alfa de Cronbach) foram: 0,83 para a escala geral; 0,89 para a escala de extroversão; 0,92 para a escala de psicoticismo. Por sua vez, a escala de neuroticismo apresentou 0,76; e, finalmente, a escala de adequação conseguiu um alfa de 0,78 . A precisão teste-reteste foi estudada com 214 crianças de 8 a 10 anos e os valores foram superiores a 0,86 .

O autor fornece cópia da Escala, critérios de correção e normas.

2 Alguns estudos de validade já foram realizados. Sisto, Pacheco, Guerrero e Urquijo (2001), utilizando o Teste das Cores de Lüscher, encontraram associação de ansiedade e tensão com neuroticismo e psicoticismo, pois crianças com traços marcantes de neuroticismo tenderam a apresentar menos tensões e ansiedades e, inversamente, as crianças com traços marcantes de psicoticismo tenderam a apresentar níveis altos de tensão e ansiedade. Por sua vez, Pacheco e Sisto (no prelo) estudando a relação entre traços de personalidade e aprendizagem por conflito soéciocognitivo e explicaram a aprendizagem no pós-teste imediato pelos traços psicoticismo e adequação. 


\section{Procedimentos}

Os instrumentos foram aplicados coletivamente, em sala de aula, por uma equipe com dois aplicadores por sala de aula, um para dar as instruções e o outro para auxiliar os sujeitos individualmente, caso fosse necessário. O Bender foi aplicado seguindo as instruções do manual. Para a Escala de Personalidade para Crianças cada criança possuía o instrumento com os itens e as alternativas (sim-não) e o aplicador lia cada item e esperava que as crianças anotassem sua resposta, fazendo um x na alternativa escolhida, após um breve treinamento para tal.

\section{RESULTADOS}

Os resultados estão apresentados em quatro blocos. No primeiro deles, apresentam-se os dados relacionados ao teste gestáltico visomotor Bender; no segundo, os dados relativos à distribuição dos traços de personalidade no grupo estudado; no terceiro, a relação entre as informações desses dois grupos de dados; e, por fim, verificou-se até que ponto os grupos extremos em relação aos traços de personalidade são discriminados quanto às medidas de integração e distorção.

\section{Teste gestáltico Visomotor Bender}

No caso da distorção, as pontuações tinham a possibilidade de variar entre 0 e 5 pontos. Observou-se que as pontuações em distorção concentraram-se em 0 , 1 e 2, congregando 76,5\% dos sujeitos estudados $(\mathrm{n}=344)$. As pontuações 3,4 e 5 foram obtidas por $23,5 \%$ das crianças. A média foi de 1,61, com desviopadrão de 1,26. Esses dados indicaram que a maioria dos sujeitos apresentou pouca distorção, e casos de distorção acentuada (pontuações 4 e 5 ) foram constatados em 28 crianças, ou $8,1 \%$ delas.

No caso da integração, as pontuações tinham a possibilidade de variar entre 0 e 7 pontos. Observou-se que as pontuações em integração concentraram-se em 0 e 1 , congregando $82,6 \%$ dos sujeitos estudados $(\mathrm{n}=344)$. As pontuações 2,3 e 4 foram obtidas por $17,4 \%$ das crianças. A média foi de 0,77 , com desviopadrão de 0,89 . Esses dados indicaram que a maioria dos sujeitos apresentou boa integração e casos de nãointegração mais acentuada (pontuações 3 e 4) foram constatados em 19 crianças, ou 5,5\% delas. Levandose em conta que a pontuação máxima seria de 7 pontos, pôde-se considerar que mesmo as mais altas pontuações detectadas nesta pesquisa foram baixas.

Com vistas a um indicador mais global, as pontuações de distorção e integração foram somadas, constituindo-se em apenas um indicador. Nesse caso, as pontuações tinham a possibilidade de variar entre 0 e 12 pontos. Observou-se que as pontuações concentraram-se em 0, 1, 2 e 3, congregando 74,7\% dos sujeitos estudados $(n=344)$. As pontuações 6,7 e 9 foram obtidas por $4,3 \%$ das crianças. A média foi de 2,38, com desvio-padrão de 1,77. Esses dados indicaram que a maioria dos sujeitos apresentou um desempenho indicativo de boa configuração, e casos com indicação de algum problema (pontuações 6,7 e 9) foram constatados em 15 crianças, ou 4,3\% delas. Tendo-se em vista que a pontuação máxima seria de 12 pontos, pôde-se considerar que mesmo as mais altas pontuações detectadas nesta pesquisa foram baixas, exceção feita à pontuação 9, obtida por um sujeito.

\section{Traços de personalidade}

A Escala de Personalidade Infantil fornece informação a respeito de quatro traços de personalidade. Em relação ao traço de personalidade neuroticismo, houve uma alta concentração entre as pontuações 5 e 8 , responsáveis por $59,8 \%$ das condutas dos sujeitos. As pontuações 9 e 10 responderam por $17,8 \%$ dos sujeitos. A média do grupo foi de 6,20 e o desvio-padrão, de 2,31. Esses dados sugeriram que o grupo estudado apresentou esse traço com uma intensidade razoavelmente alta.

Em relação ao traço extroversão, também se encontrou uma alta concentração em pontuações altas, pois os sujeitos com 7,8 e 9 pontos perfizeram $81 \%$ dos protocolos estudados. Com uma média de 7,83 e um desvio-padrão de 1,34 pontos, a extroversão foi o traço com a maior média em relação aos outros traços.

$\mathrm{O}$ traço de psicoticismo, por sua vez, apresentou uma concentração bastante acentuada na pontuação zero $(34,3 \%)$, e as pontuações zero, um e dois explicaram $63 \%$ das condutas dos sujeitos. A pontuação máxima alcançada foi 8 , e $9,7 \%$ dos sujeitos tiveram uma pontuação entre 5 e 8 . A média foi de 1,53 e o desvio-padrão, de 1,94. Esse tipo de distribuição parece caracterizar esse traço.

As pontuações entre 2 e 6 na escala de adequação abarcaram quase todos os resultados e explicaram $83,5 \%$ das condutas. Houve certa concentração nas pontuações 3 e 4 (32\%). A média encontrada foi de 3,55 e o desvio-padrão, de 1,79 pontos. Esses dados indicaram uma boa distribuição do traço nos sujeitos estudados.

\section{Relação entre traços de personalidade e integração e distorção de forma.Integração}

Considerando-se que, nos itens 2 e 5 , apenas um sujeito obteve pontuação 1 e no item 3,8 sujeitos 
obtiveram a pontuação 1, eles foram eliminados para calcular as correlações; no entanto, eles foram mantidos quando a medida foi a soma de itens.

Tabela 1. Coeficientes de correlação de Pearson entre traços de personalidade e medidas de integração.

\begin{tabular}{lcccc}
\hline & \multicolumn{4}{c}{ Traços de Personalidade } \\
\cline { 2 - 5 } Medidas do Bender & NeuroticismoExtroversão Psicoticismo Sinceridade \\
\hline integração & $0,182 * * * *$ & 0,080 & 0,106 & $-0,001$ \\
figura 7 & $0,170 * * *$ & 0,055 & 0,044 & $-0,012$ \\
figura 3 & $0,166 * *$ & 0,083 & $0,212 * * * * *$ & 0,051 \\
integração & $0,218 * * * * *$ & 0,102 & $0,142 *$ & 0,018 \\
acentuada & 0,052 & $-0,008$ & $-0,035$ & 0,024 \\
figura A & 0,085 & 0,087 & 0,037 & $-0,046$ \\
figura 4 & $-0,096$ & 0,003 & $-0,112$ & $-0,065$ \\
figura 5 & * & & & \\
\hline p $=0,017$ & $* * \mathrm{p}=0,005 * * \mathrm{p}=0,004$ & $* * * * \mathrm{p}=0,002$ & $* * * * * \mathrm{p}=0,000$ &
\end{tabular}

Com relação à integração, $o$ traço de personalidade neuroticismo foi o que produziu maior número de correlações significativas, pois elas ocorreram nos dois indicadores constituídos por somas de itens (integração e integração acentuada) e em 2 dos 6 itens individuais (figura 7 e figura 3), todas elas positivas, conforme pode ser observado na Tabela 1 . Em duas situações o traço psicoticismo teve correlações positivas e significativas, quais sejam, figura 3 e integração acentuada. Deve-se ressaltar que as medidas figura 3 e integração mantiveram correlações positivas e significativas tanto com o neuroticismo quanto com o psicoticismo.

Considerando-se que as altas pontuações nas medidas do Bender indicaram uma menor integração e que as altas pontuações na Escala de Personalidade Infantil indicaram maior presença do traço em questão, esses resultados sugeriram que, conforme diminuiu a integração, aumentou a intensidade do traço em questão. Nesse contexto, pôde-se inferir que a integração aferida pelo teste gestáltico Bender manteve uma certa associação com o traço de neuroticismo, principalmente nas duas medidas produtos de somas dos itens; além disso, o psicoticismo também apresentou correlação significativa com uma das medidas globais, sugerindo que a não-integração também faz parte do quadro de psicoticismo.

\section{Distorção}

A Tabela 2 apresenta os resultados das correlações entre distorção das figuras e traços de personalidade. Diferentemente do caso da integração, nenhum dos itens avaliados apresentou problemas de discriminação, não sendo eliminado nenhum para efeitos de análise.
Tabela 2. Coeficientes de correlação de Pearson entre traços de personalidade e medidas de distorção.

Traços de Personalidade Medidas do Bender Neuroticismo Extroversão Psicoticismo Sinceridade

\begin{tabular}{lcccc}
\hline figura A & 0,048 & 0,106 & $0,219 * * *$ & 0,041 \\
figura 7 & 0,088 & 0,052 & $0,142 *$ & $-0,114$ \\
figuras A e 7 & 0,093 & 0,103 & $0,241^{* * *}$ & $-0,055$ \\
distorção de forma & 0,073 & 0,070 & $0,166 * *$ & $-0,045$ \\
figura 1 & 0,030 & $-0,062$ & $-0,083$ & $-0,069$ \\
figura 3 & $-0,005$ & $-0,004$ & $-0,043$ & 0,028 \\
figura 5 & $-0,046$ & $-0,045$ & $-0,085$ & 0,022 \\
Distorção & 0,037 & 0,021 & 0,055 & $-0,025$ \\
ponderada & & & $-0,092$ & 0,005 \\
figuras 1, 3 e 5 & $-0,018$ & $-0,044$ & & \\
* p $=0,017$ ** $\mathrm{p}=0,005$ & $* * * \mathrm{p}=0,000$ & & &
\end{tabular}

As medidas de distorção do teste gestáltico de Bender apresentaram correlações significativas apenas com o traço psicoticismo, todas elas positivas, em quatro das 9 medidas, quais sejam, figura A, figura 7, figuras A e 7 e distorção de forma. Considerando-se que as pontuações do Bender aumentaram conforme a característica da distorção constatada, pôde-se inferir que, quanto maior a distorção e quanto mais vezes elas foram registradas, maior foi a intensidade do traço psicoticismo.

\section{Discriminação das medidas}

Com vistas a estudar a discriminação das medidas, separaram-se os dois grupos de pontuações extremas em relação aos traços de personalidade, por meio das medidas fornecidas pela padronização do instrumento. Considerando-se os itens individualmente, as diferenças das médias dos grupos extremos em relação à distorção foram analisadas pela prova $t$ de student e seus resultados encontram-se na Tabela 3.

Tabela 3. Resultados $t$ entre grupos extremos em traços de personalidade e itens de distorção.

\begin{tabular}{lcccc}
\hline & \multicolumn{5}{c}{ Traços de Personalidade } \\
\cline { 2 - 6 } Medidas do Bender & Neuroticismo Psicoticismo & Adequação Extroversão \\
\hline figura A & $-0,908$ & $-3,570^{* *}$ & 0,000 & $-1,410$ \\
figura 1 & $-0,747$ & 1,143 & 1,109 & 1,819 \\
figura 3 & $-0,130$ & $-0,134$ & $-1,002$ & 0,000 \\
figura 5 & 0,574 & 0,865 & 0,367 & 1,304 \\
figura 7 & $-0,932$ & $-2,114 *$ & 1,283 & 0,251 \\
\hline * $=0,036$ & $* * \mathrm{p}=0,000$ & \multicolumn{5}{c}{}
\end{tabular}

Apenas dois itens relativos à distorção medida pela Bender discriminaram os grupos extremos criados com base nos traços de personalidade, ambos relacionados ao psicoticismo, quais sejam figura $\mathrm{A} e$ figura 7. Os resultados dos outros itens sugeriram pouca discriminação desses grupos. Esses dados 
alicerçariam um pouco mais a hipótese de que a distorção da figuras caracterizaria crianças com traços de psicoticismo.

Considerando-se os itens individualmente, as diferenças das médias dos grupos extremos em relação à integração foram analisadas pela prova $t$ de student $\mathrm{e}$ seus resultados encontram-se na tabela 4 .

Tabela 4. Resultados $t$ entre grupos extremos em traços de personalidade e itens de integração.

Traços de Personalidade

Medidas do Bender Neuroticismo Psicoticismo Adequação Extroversão

\begin{tabular}{lcccc}
\hline figura A & $-0,094$ & 1,086 & 0,147 & $-0,537$ \\
figura 3 & $-2,251 * *$ & $-3,114 * * * *$ & $-1,002$ & $-0,887$ \\
figura 4 & $-1,023$ & $-0,241$ & $-0,659$ & 0,599 \\
figura 5 & $2,097 *$ & 1,636 & $-0,535$ & $-0,411$ \\
figura 7 & $-2,339 * * *$ & $-0,606$ & $-0,460$ & 0,263 \\
\hline
\end{tabular}

$* \mathrm{p}=0,0,039 * * \mathrm{p}=0,027 * * * \mathrm{p}=0,022 * * * * \mathrm{p}=0,002$

Os itens relacionados à integração medida pelo Bender apresentaram mais resultados significativos no que se refere à discriminação que os de distorção. Além disso, não discriminaram apenas um traço de personalidade, como foi o caso anterior. Assim, os itens figura 3 , figura 5 e figura 7 discriminaram significativamente os grupos extremos de neuroticismo. O item figura 3, além de neuroticismo, discriminou bastante bem os grupos extremos de psicoticismo.

Foram analisadas também as medidas com base na soma de itens. A tabela 5 apresenta os resultados tanto das somas de itens de integração quanto de distorção.

Tabela 5. Resultados $t$ entre grupos extremos em traços de personalidade e medidas produtos da soma de itens.

\begin{tabular}{lcccc}
\hline & \multicolumn{4}{c}{ Traços de Personalidade } \\
\cline { 2 - 5 } Medidas do Bender & NeuroticismoPsicoticismo & Adequação Extroversão \\
\hline integração & $-2,275^{*}$ & $-1,233$ & $-1,129$ & 0,079 \\
integração acentuada & $-2,830 * *$ & $-1,289$ & 0,466 & 0,461 \\
distorção ponderada & $-1,013$ & $-2,773 * *$ & 0,718 & $-0,232$ \\
figuras A e 7 & $-1,157$ & $-3,735 * * *$ & 0,819 & $-0,755$ \\
distorção & $-0,678$ & $-1,289$ & 0,466 & 0,461 \\
geral & $-1,535$ & $-1,568$ & $-0,155$ & 0,345 \\
figuras 1, 3 e 5 & 0,025 & 0,761 & 0,000 & 1,148 \\
\hline$* \mathrm{p}=0,025$ & $* * \mathrm{p}=0,006$ & $* * * \mathrm{p}=0,000$ & &
\end{tabular}

$* \mathrm{p}=0,025 \quad * * \mathrm{p}=0,006 \quad * * * \mathrm{p}=0,000$

Os resultados das análises estatísticas das pontuações advindas da soma de itens utilizadas como medida para discriminar os grupos extremos formados pelos traços de personalidade revelaram principalmente dois fatos. O primeiro deles foi que as duas medidas de integração (integração e integração abreviada) discriminaram os grupos extremos de neuroticismo e duas das medidas de distorção (figuras
A e 7; e distorção ponderada) discriminaram os grupos extremos de psicoticismo. O segundo deles foi que os grupos extremos de adequação e extroversão não puderam ser discriminados por nenhuma das medidas usadas e a soma das pontuações de integração e distorção (geral) também não discriminou grupo algum.

\section{CONCLUSÕES (OU À GUISA DE CONCLUSÃO)}

Este estudo foi proposto com base na afirmação de Bender (1938) de que a qualidade da reprodução das figuras pelas crianças estaria determinada por fatores biológicos e de ação sensório-motora, cuja variação estaria em razão tanto de seu padrão de desenvolvimento e nível maturacional como de seu estado patológico funcional e organicamente induzido.

Os dados encontrados sugerem que o traço de personalidade neuroticismo parece estar mais associado à medida relacionada à integração, pois se correlacionou com quatro das sete medidas. $\mathrm{O}$ traço psicoticismo também teve correlações positivas e significativas com duas das medidas de integração, sendo que uma delas também se correlacionou com neuroticismo. A tendência encontrada indicou que, conforme diminuiu a integração, aumentou a intensidade do traço em questão. Por sua vez, a medida de distorção do teste gestáltico de Bender associou-se positiva e significativamente apenas com o traço psicoticismo, podendo-se inferir que, quanto mais distorções nas figuras, maior seria a intensidade do traço psicoticismo.

Para saber se as medidas de distorção e integração discriminariam grupos extremos por traços de personalidade e considerando-se os itens individualmente, observou-se que, com relação à distorção, apenas dois itens apresentaram significância estatística, ambos relacionados ao psicoticismo. Esses dados pareceram alicerçar um pouco mais a hipótese de que a distorção das figuras caracterizaria crianças com traços de psicoticismo. No que concerne aos itens relacionados à integração, mais itens apresentaram significância estatística, sendo que três itens discriminaram significativamente os grupos extremos de neuroticismo e um item discriminou bastante bem os grupos extremos de psicoticismo. Quando as medidas foram a soma de itens, duas medidas de integração discriminaram os grupos extremos de neuroticismo e duas das medidas de distorção discriminaram os grupos extremos de psicoticismo.

As análises feitas sugeriram principalmente quatro regularidades de resultados. Um deles foi a associação entre a medida de integração e neuroticismo. O 
segundo, a relação entre distorção e psicoticismo. $\mathrm{O}$ terceiro, que há uma intersecção entre os constructos psicoticismo e o neuroticismo, observado pela medida relacionada à integração. $\mathrm{E}$, finalmente, os traços de personalidade adequação e extroversão não se relacionaram com as medidas de integração e distorção, conforme avaliados pelo teste gestáltico visomotor de Bender.

O modelo fatorial da personalidade tem incluído três dimensões tipológicas básicas: introversão versus extroversão, neuroticismo versus estabilidade emocional e psicoticismo versus controle dos impulsos. As diferentes interações entre essas três dimensões estruturariam as diferenças individuais de temperamento e cada fator poderia ser identificado isoladamente por meio de análise fatorial. Uma dimensão tipológica básica se refere a um traço de personalidade que tem sido considerado como um conjunto de comportamentos relacionados que covariam ou ocorrem juntos repetidamente; enquanto um tipo seria um construto de ordem superior, compreendendo um conjunto de traços correlacionados.

Alguns aspectos de condutas têm caracterizado as pessoas com altas pontuações em neuroticismo. Sua descrição, em geral, é dada em termos de comportamentos emocionais, pois considerado extremamente emotivo, tais como ansioso, rabugento, freqüentemente deprimido e preocupado. Em razão de apresentar reações emocionalmente exageradas aos estímulos e ter dificuldades para acalmar-se, geralmente não se caracteriza por um ajustamento adequado, muitas vezes reagindo de maneira irracional e às vezes rígida. É interessante observar que crianças com essas características apresentaram também grandes dificuldades em integrar elementos básicos de figuras bastante simples e primitivas. A não-integração de dados básicos das situações não deixa de ser um componente interessante em um quadro de dificuldades de controle emocional e de reações fortes e emotivas. Ao se acrescentar que uma pessoa com elevado grau de neuroticismo tem como principal característica a preocupação com ações e fatos que poderiam dar errado, não fica difícil perceber que, se ela tem dificuldade em integrar elementos básicos, a chance de errar em suas previsões não deixa de ser alta, e a forte reação emocional de ansiedade diante de situações novas poderia estar ancorada em contínuas situações de não conseguir prever e controlar o inusitado, pois inábil para integrar os elementos constituintes do contexto.

$\mathrm{O}$ quadro psicológico da criança com altas pontuações em psicoticismo é bastante diferente em relação à criança com altas pontuações em neuroticismo. Normalmente é tida como isolada, fria, estranha e com sentimentos poucos humanos, tanto para com as pessoas quanto para com os animais. É uma pessoa perturbadora, muitas vezes descrita como agressiva e hostil, mesmo no relacionamento com pessoas próximas e supostamente queridas, reforçando a idéia de falta de sentimentos. Busca o excesso, o excitante, o perigo, e parece não ter medo deles. De socialização difícil, a falta de sentimentos de culpa e sensibilidade em relação ao outro parece fazer parte do quadro das crianças com altas pontuações em psicoticismo. Ao lado disso, pelos dados desta pesquisa há também uma forte tendência a distorcer figuras simples, mudar a forma de elementos estruturados e simples e uma pequena tendência a não integrar os elementos dessas mesmas figuras. $\mathrm{O}$ distorcer e não integrar elementos de uma estrutura simples estar presentes junto a condutas de frieza e falta de sentimentos, mesmo com os seus entes mais próximos e com animais de seu convívio, suscita a discussão de que sentimentos e perigo são aspectos aceitos como produto de uma aprendizagem, aprendizagem essa que estaria prejudicada em seus aspectos mais internos, se estruturalmente a pessoa em questão tende a distorcer a forma das relações observadas. É uma hipótese que não deixa de ser interessante considerar.

A literatura investigada tende a mostrar uma relação às vezes, não muito contundente, entre os resultados do teste Bender e quadros psicopatológicos. Mas o acúmulo de pesquisas leva a crer que, ainda embora de forma não muito precisa, esses elementos estruturais gestálticos parecem guardar relações com sintomas psicopatológicos. Parece que isso não ocorre apenas com pessoas já clinicamente diagnosticadas, mas também com traços de personalidade de pessoas que, aparentemente, não apresentam quadros psicopatológicos clínicos.

\section{REFERÊNCIAS}

Archer, R. P., Maruish, M., Imhof, E. A., e Piotrowski, C. (1991). Psychological test usage with adolescent clients: 1990 survey findings. Professional Psychology: Research and Practice, 22(3), 247-252.

Bambaren-Vigil, C. (1958). La prueba de la "gestalt" de bender, en esquizofrénicos. Revista de Psicología General y Aplicada, 13, 481-509.

Bender, L. (1938). A visual-motor gestalt test and its clinical use. The American Orthopychiatric Association, Research Monograph n $\mathbf{0} 3$.

Brannigan, G. G., Barone, R.J., e Margolis, H. (1978). Bender gestalt signs as indicants of conceptual impulsivity. Journal of Personality Assessment, 42(3), 233-236. 
Brilliant, P. J., \& Gynther, M.D. (1963). Relationships between performance on three tests for organicity and selected patient variables. Journal of Consulting Psychology, 27(6), 474-479.

Clawson, A. (1980). Bender infantil. Porto Alegre: Artes Médicas.

Gavales, D., \& Millon, T. (1960). Comparison of reproduction and recall size deviations in the bender gestalt as measures of anxiety. Journal of Clinical Psychology, 16, 278-280.

Handler, L., \& McIntosh, J. (1971). Predicting aggression and withdrawal in children with the draw a person and bender gestalt. Journal of Personality Assessment, 35(4), 331-335.

Holmes, C. B., Dungan, D.S. e Medlin, W. J. (1984). Reassessment of inferring personality traits from Bender Gestalt drawing styles. Journal of Clinical Psychology, 40 (5), 1241-1243.

Hutt, M. L., Dates, B.G. e Reid, D.M. (1977). The predictive ability of HABGT scales for a male delinquent population. Journal of Personality Assessment, 41(5), 492-496.

Hutt, M. L. (1985). The hutt adaptation of the bender-gestalt test. Orlando: Grune \& Stratton.

Johnson, J. H. (1973). Bender gestalt constriction as an indicator of depression in psychiatric patients. Journal of Personality Assessment, 37(1), 53-55.

Kamman, G. R. \& Kram, C. (1955). Value of psychometric examinations in medical diagnosis and treatment. Journal of the American Medical Association, 158, 555-560.

Koppitz, E. M. (1964). The bender gestalt test for young children. Orlando: Grune, \& Stratton.

Korin, H. (1974). Comparison of psychometric measures in psychiatric patients using heroin and other drugs. Journal of Abnormal Psychology, 83(2), 208-212.

Lacks, P. (1984). Bender gestalt screening for brain damage dysfunction. New York: Wiley \& Sons.

Lownsdale, W. S., Rogers, B. J. e McCall, J. N. (1989). Concurrent validation of hutt's bender gestalt screening method for schizophrenia, depression, and brain damage. Journal of Personality Assessment, 53(4), 832-836.

Matchabely, K. \& Bertrand, R. (1953). Quelques considerations pratiques sur l'application du test moteur de structuration visuelle de bender en clinique psychiatrique: some practical considerations on the application of Bender's Visual Motor Gestalt test in clinical psychiatry. Revue de Psychologie Appliquee, 3, 326-332.

Murray, E. J., \& Roberts, F. J. (1956). The bender gestalt test in a patient passing through a brief manic depressive cycle. United States Armed Forces Medical Journal, 7, 1206-1208.

Pascal, G. \& Suttell, B. (1951). The bender gestalt-test. New York: Grune \& Stratton.

Porterfield, C.L. (1969). Adaptive mechanisms of young disadvantaged stutterers and nonstutterers. Journal of
Projective Techniques and Personality Assessment, 33(4), 371-375.

Prado, W. M., Peyman, D.A. \& Lacey, O. L. (1960). A validation study of measures of flattened affect on the Bender Gestalt Test. Journal of Clinical Psychology, 16(4), 435-438.

Rossini, E.D. (1993). The bender gestalt psychopathology scale: failure to infer validity in a school aged sample. Journal of Personality Assessment, 60 (3), 605.

Sangster, G., Rogers, B.J. \& Searight, H.R. (1993). The validity of Hutt's Bender Gestalt scoring system for hostility: correlation with the interpersonal behavior survey's aggression scales. Psychology: A Journal of Human Behavior, 30 (2), 22-29

Sisto, F.F. (1998). Escala de Traços de Personalidade para Crianças. Unicamp, Departamento de Psicologia Educacional.

Sisto, F. F., Pacheco, L. M. B., Guerrero, P. V. T. e Urquijo, S. (2001). La tensión y la ansiedad en los rasgos de personalidad: un estudio exploratorio de validación. Acta Psiquiátrica y Psicológica de América Latina, 47(4), 340350.

Pacheco, L. \& Sisto, F. F. (no prelo). Aprendizagem por interação e traços de personalidade. Revista Psicologia Escolar e Educacional.

Sternberg, D. \& Levine, A. (1965). An indicator of suicidal ideation on the bender visual motor gestalt Test. Journal of Projective Techniques and Personality Assessment, 29, 377 379.

Stewart, H., \& Cunningham, S. (1958). A note on scoring recalled figures of the Bender Gestalt Test using psychotics, non psychotics and controls. Journal of Clinical Psychology, 14, 207-208.

Tamkin, A. S. (1957). The effectiveness of the bender gestalt in differential diagnosis. Journal of Consulting Psychology, 21,355-357.

Wallbrown, F. H., Wirth, E., e Engin, A.W. (1975). Some evidence relevant to Kagan's comments on the clinical interpretation of the bender gestalt. Journal of Clinical Psychology, 31(2), 345-347.

Wasserman, C. B. (1995). A study of the perceptual adienceabience scale of the hutt adaptation of the bender-gestalt test. Dissertation Abstracts International Section A: Humanities and Social Sciences, 55 (12-A), 3752.

Wohl, J. (1957). A note on the generality of constriction. Journal of Projective Techniques, 21, 410-413.

Recebido em 27/01/2003

Revisado em 27/05/2003

Aceito em 30/05/2003 